\title{
Adaptive State-Feedback Stabilization for Stochastic Nonholonomic Mobile Robots with Unknown Parameters
}

\author{
Wenli Feng, ${ }^{1}$ Qingli Sun, ${ }^{1}$ Zhijun Cao, ${ }^{1}$ Dongkai Zhang, ${ }^{1}$ and Hua Chen ${ }^{2}$ \\ ${ }^{1}$ School of Mathematics and Information Science, Shijiazhuang University, Hebei 050035, China \\ ${ }^{2}$ Department of Mathematics and Physics, Changzhou Campus, Hohai University, Jiangsu 213022, China
}

Correspondence should be addressed to Dongkai Zhang; zdkmailhot@126.com

Received 19 September 2013; Accepted 29 October 2013

Academic Editor: Guoliang Wei

Copyright (C) 2013 Wenli Feng et al. This is an open access article distributed under the Creative Commons Attribution License, which permits unrestricted use, distribution, and reproduction in any medium, provided the original work is properly cited.

The stabilizing problem of stochastic nonholonomic mobile robots with uncertain parameters is addressed in this paper. The nonholonomic mobile robots with kinematic unknown parameters are extended to the stochastic case. Based on backstepping technique, adaptive state-feedback stabilizing controllers are designed for nonholonomic mobile robots with kinematic unknown parameters whose linear velocity and angular velocity are subject to some stochastic disturbances simultaneously. A switching control strategy for the original system is presented. The proposed controllers that guarantee the states of closed-loop system are asymptotically stabilized at the zero equilibrium point in probability.

\section{Introduction}

In the past decades, the control of nonholonomic systems has been widely pursued. By the results of Brockett [1], the nonholonomic system cannot be stabilized at a single equilibrium point by any static smooth pure state-feedback controller. To solve this problem, lots of novel approaches have been considered: discontinuous feedback control [2-4], smooth time-varying feedback controller [5], and the method of LMI [6]. The control of nonholonomic mobile robots plays an important role in that of nonholonomic systems because they are a benchmark for these systems. There is much attention devoted to the control of nonholonomic mobile robots. The nonholonomic mobile robots were classified into four types, which were characterized by generic structures of the model equations [7]. Based on the backstepping technique, the control for nonholonomic mobile robots was discussed: tracking problems [8] and stabilizing problems $[9,10]$. Hespanha et al. introduced the mobile robot with parametric uncertainties [11], which were further discussed $[12,13]$. But all the above articles discussed the nonholonomic systems in the deterministic case, which was not considered a stochastic disturbance.
In recent years, stochastic nonlinear systems have received much attention $[14,15]$, especially for stochastic control when backstepping designs were firstly introduced [16, 17]. For stochastic nonholonomic systems, there were a few papers. The almost global adaptive asymptotical controllers of stochastic nonholonomic chained form systems were discussed by using discontinuous control [18]. The adaptive stabilization problem of stochastic nonholonomic systems with nonlinear drifts was considered [19-21]. By using statescaling method, backstepping controllers were proposed to deal with exponential stabilization for nonholonomic mobile robots with stochastic disturbance [22,23]. But the above two papers did not consider unknown parameters. To our knowledge, the problem of adaptive state-feedback stabilization for nonholonomic mobile robots with kinematic unknown parameters, whose linear velocity and angular velocity are subject to some stochastic disturbances simultaneously, has not been reported. So, there exists a natural problem which is how to extend the models in [11-13] to the stochastic case and design an adaptive state-feedback stabilizing controller for stochastic nonholonomic mobile robots with uncertain parameters. 
The purpose of this paper is to design adaptive statefeedback stabilizing controllers for stochastic nonholonomic mobile robots with unknown parameters. The main idea of this paper is highlighted as follows.

(i) We extend the models of nonholonomic mobile robots with unknown parameters in [11-13] to the stochastic case. The stabilizing controllers are designed for stochastic nonholonomic mobile robots with unknown parameters by adaptive state-feedback backstepping technique.

(ii) A switching control strategy for the original system is presented. It guarantees that the states of closedloop system are asymptotically stabilized at the zero equilibrium point in probability.

The paper is organized as follows. Section 1 begins with the mathematical preliminaries. In Section 2, the adaptive state-feedback backstepping controller is designed. In Section 3, a switching control strategy for the original system is discussed. Finally, a simulation example is given to show the effectiveness of the controller in Section 4.

\section{Preliminaries and Problem Formulation}

2.1. Preliminaries. Consider the following stochastic nonlinear system:

$$
\mathrm{d} x=f(x) \mathrm{d} t+g(x) \mathrm{d} B, \quad x\left(t_{0}\right) \in \mathbb{R}^{n},
$$

where $x \in \mathbb{R}^{n}$ is the state, the Borel measurable functions $f$ : $\mathbb{R}^{n} \rightarrow \mathbb{R}^{n}$ and $g: \mathbb{R}^{n} \rightarrow \mathbb{R}^{n \times r}$ are locally Lipschitz in $x$, and $B \in \mathbb{R}^{r}$ is an $r$-dimensional independent standard Wiener process defined on the complete probability space $(\Omega, \mathscr{F}, P)$.

The following definitions and lemmas will be used in the paper.

Definition 1 (see [16]). For any given $V(x) \in \mathscr{C}^{2}$, associated with stochastic system (1), the differential operator $\mathscr{L}$ is defined as follows:

$$
\mathscr{L} V(x)=\frac{\partial V}{\partial x} f(x)+\frac{1}{2} \operatorname{Tr}\left\{g^{T}(x) \frac{\partial^{2} V}{\partial x^{2}} g(x)\right\} .
$$

Definition 2 (see [24]). The equilibrium $x=0$ of system (1) is

(i) globally stable in probability if for $\forall \varepsilon>0$, there exists a class $\mathscr{K}$ function $\gamma(\cdot)$ such that

$$
P\left\{|x(t)|<\gamma\left(\left|x\left(t_{0}\right)\right|\right)\right\} \geq 1-\varepsilon, \quad \forall t \geq 0, x\left(t_{0}\right) \in \mathbb{R}^{n} \backslash\{0\} ;
$$

(ii) globally asymptotically stable in probability if it is globally stable in probability and

$$
P\left\{\lim _{t \rightarrow \infty}|x(t)|=0\right\}=1, \quad \forall x\left(t_{0}\right) \in \mathbb{R}^{n} .
$$

Definition 3 (see [25]). A stochastic process $x(t)$ is said to be bounded in probability if the random variable $|x(t)|$ is bounded in probability uniformly in $t$; that is,

$$
\lim _{R \rightarrow \infty} \sup _{t>t_{0}} P\{|x(t)|>R\}=0 .
$$

Lemma 4 (see [24]). Considering the stochastic system (1), if there exist a $\mathscr{C}^{2}$ function $V(x)$, class $\mathscr{K}_{\infty}$ functions $\alpha_{1}(\cdot)$ and $\alpha_{2}(\cdot)$, constants $c_{1}>0, c_{2} \geq 0$, and a nonnegative function $W(x)$ such that

$$
\begin{gathered}
\alpha_{1}(|x|) \leq V(x) \leq \alpha_{2}(|x|), \\
\mathscr{L} V(x)=\frac{\partial V}{\partial x} f+\frac{1}{2} \operatorname{Tr}\left\{g^{T} \frac{\partial^{2} V}{\partial x^{2}} g\right\} \\
\leq-c_{1} W(x)+c_{2},
\end{gathered}
$$

then

(i) for (1), there exists an almost surely unique solution on $\left[t_{0}, \infty\right)$ for each $x\left(t_{0}\right) \in \mathbb{R}^{n}$;

(ii) when $c_{2}=0, f(0)=0, g(0)=0$, and $W(x)$ is continuous, then the equilibrium $x=0$ is globally stable in probability and

$$
P\left\{\lim _{t \rightarrow \infty} W(x(t))=0\right\}=1, \quad \text { for } \forall x\left(t_{0}\right) \in \mathbb{R}^{n} .
$$

Lemma 5 (see [26]). Let $x$ and $y$ be real variables. Then, for any positive integers $m, n$ and any real number $\varepsilon>0$, the following inequality holds:

$$
|x|^{m}|y|^{n} \leq \frac{m}{m+n} \varepsilon|x|^{m+n}+\frac{n}{m+n} \varepsilon^{-m / n}|y|^{m+n} .
$$

2.2. Problem Formulation. Hespanha et al. introduced the mobile robot with parametric uncertainties [11], which were further discussed in $[12,13]$ as follows:

$$
\begin{gathered}
\dot{\theta}=p_{1}^{*} \omega, \quad \dot{x}_{c}=p_{2}^{*} v \cos \theta, \\
\dot{y}_{c}=p_{2}^{*} v \sin \theta,
\end{gathered}
$$

where $v$ and $\omega$ are two control inputs to denote the forward velocity and angular velocity, respectively.

Here we assume that the forward velocity $v$ and the angular velocity $\omega$ are subject to some stochastic disturbances. Based on the similar methods in [27, Page 1-2], velocity $v$ and the angular velocity $\omega$ with stochastic disturbances can be expressed as follows:

$$
\begin{gathered}
\omega(\theta)=\omega_{1}(\theta)+\omega_{2}(\theta) \dot{B}(t), \\
v\left(x_{c}, y_{c}, \theta\right)=v_{1}\left(x_{c}, y_{c}, \theta\right)+v_{2}\left(x_{c}, y_{c}, \theta\right) \dot{B}(t),
\end{gathered}
$$

where $\dot{B}(t)$ is the derivative of a Brownian motion $B(t)$.

Remark 6. The second equality of (10) is the same as that of Remark 2 in [19]. Moreover, (10) means that $\omega(\theta)$ can be divided into two parts, with the second parts being stochastic disturbances and the same for $v\left(x_{c}, y_{c}, \theta\right)$.

Substituting (10) into (9), the system (9) can be transformed into

$$
\begin{aligned}
\mathrm{d} \theta & =p_{1}^{*} \omega_{1} \mathrm{~d} t+p_{1}^{*} \omega_{2} \mathrm{~d} B \\
\mathrm{~d} x_{c} & =p_{2}^{*} v_{1} \cos \theta \mathrm{d} t+p_{2}^{*} v_{2} \cos \theta \mathrm{d} B, \\
\mathrm{~d} y_{c} & =p_{2}^{*} v_{1} \sin \theta \mathrm{d} t+p_{2}^{*} v_{2} \sin \theta \mathrm{d} B
\end{aligned}
$$


where $p_{1}^{*}$ is unknown parameter taking values in a known interval $\left[p_{\min }, p_{\max }\right]$ with $0<p_{\min }<p_{\max }<\infty$; $p_{2}^{*}$ is unknown positive parameter.

For system (11), we introduce the following state and input transformation:

$$
\begin{aligned}
& x_{0}=\theta, \quad u_{0}=\omega_{1}, \quad u=v_{1}, \\
& x_{1}=x_{c} \sin \theta-y_{c} \cos \theta, \\
& x_{2}=x_{c} \cos \theta+y_{c} \sin \theta,
\end{aligned}
$$

and it is easy to see that

$$
\begin{aligned}
\mathrm{d} x_{0}= & p_{1}^{*} u_{0} \mathrm{~d} t+p_{1}^{*} \omega_{2}\left(x_{0}\right) \mathrm{d} B, \\
\mathrm{~d} x_{1}= & p_{2}^{*} x_{2} u_{0} \mathrm{~d} t-\frac{1}{2}\left(p_{1}^{*}\right)^{2} x_{1} \omega_{2}^{2} \mathrm{~d} t \\
& +p_{1}^{*} p_{2}^{*} v_{2} \omega_{2} \mathrm{~d} t+p_{1}^{*} x_{2} \omega_{2} \mathrm{~d} B, \\
\mathrm{~d} x_{2}= & p_{2}^{*} u \mathrm{~d} t-\left(p_{1}^{*} x_{1} u_{0}+\frac{1}{2}\left(p_{1}^{*}\right)^{2} x_{2} \omega_{2}^{2}\right) \mathrm{d} t \\
& +\left(p_{2}^{*} v_{2}-p_{1}^{*} x_{1} \omega_{2}\right) \mathrm{d} B .
\end{aligned}
$$

Remark 7. The main difference between this paper and [22] is that the unknown parameter exists in this paper. The controller design of systems (13a) and (13b) will be more difficult.

Remark 8. For system (13a) and (13b), the variable $x_{2}$ appears in the term $p_{1}^{*} x_{2} \omega_{2} \mathrm{~d} B$ in the first equation of (13b); this is different from the traditional stochastic backstepping technique in $[16,17,24]$.

\section{Adaptive Controller Design}

In this section, we will design state-feedback controllers such that all the signals in closed-loop system are regulated to the origin in probability. The following assumptions are needed.

Assumption 9. For the smooth function $\omega_{2}(\theta)$, there exists a known positive constant $m_{1}$, such that

$$
\omega_{2}(\theta)=m_{1} \theta
$$

Assumption 10. For smooth function $v_{2}\left(x_{c}, y_{c}, \theta\right)$ and any positive constant $C$, there exists a known nonnegative constant $m_{2}$, such that

$$
\left|v_{2}\left(x_{c}, y_{c}, \theta\right)\right| \leq m_{2}\left|x_{2}-C \frac{x_{1}}{u_{0}}\right| .
$$

Remark 11. For the adaptive controllers' design in the following, if we let $C=c_{1}$, this assumption will change to $\left|v_{2}\left(x_{c}, y_{c}, \theta\right)\right| \leq m_{2}\left|\varepsilon_{2}\right|$, where $\varepsilon_{2}$ is defined in (25) and $c_{1}$ is the same as that in (28) in the following Section 3.2.

Firstly, we will consider the problem of stabilization for systems (13a) and (13b) under the condition of $x_{0}\left(t_{0}\right) \neq 0$. The case of $x_{0}\left(t_{0}\right)=0$ will be discussed in Section 3 .
3.1. The First State Stabilization. Let us consider the subsystem (13a) of stochastic nonholonomic nonlinear systems (13a) and (13b):

$$
\mathrm{d} x_{0}=p_{1}^{*} u_{0} \mathrm{~d} t+p_{1}^{*} \omega_{2}\left(x_{0}\right) \mathrm{d} B .
$$

In order to guarantee that $x_{0}$ converges to zero, one can take $u_{0}$ as follows:

$$
u_{0}=-\eta_{0} x_{0}, \quad \eta_{0}=\lambda+\frac{3}{2} m_{1}^{2} p_{\max },
$$

where $\lambda>0$ is a design parameter.

If we employ a Lyapunov function of the form:

$$
V_{0}\left(x_{0}\right)=\frac{1}{4 p_{1}^{*}} x_{0}^{4} .
$$

From (13a), (17), (18), and Assumption 9, one can obtain

$$
\mathscr{L} V_{0} \leq x_{0}^{3} u_{0}+\frac{3}{2 p_{1}^{*}} x_{0}^{2}\left(p_{1}^{*}\right)^{2} \omega_{2}^{2} \leq-\lambda x_{0}^{4}
$$

Theorem 12. If Assumption 9 holds, one can choose positive constants $m_{1}, \lambda$, and $p_{\max }$ and the controller $u_{0}$ as (17), respectively, then

(i) the closed-loop subsystem composed by (13a) and (17) has an almost surely unique solution on $\left[t_{0}, \infty\right)$ for $\forall x_{0}\left(t_{0}\right)$;

(ii) the equilibrium $x_{0}=0$ of the closed-loop subsystem composed by (13a) and (17) is globally asymptotically stable in probability.

Proof. Choosing Lyapunov function as (18), by (19), $\lambda>$ 0 , and Lemma 4, (i) holds and the equilibrium $x_{0}=0$ of the closed-loop subsystem which contained (13a) and (17) is globally stable in probability and for $\forall x_{0}\left(t_{0}\right) \neq 0$, $P\left\{\lim _{t \rightarrow \infty}\left(\left|x_{0}(t)\right|=0\right)\right\}=1$. From Definition 2, (ii) holds.

Remark 13. From Theorem 12, one has the state $x_{0}$ bounded in probability; that is, there exists a positive constant $m_{3}$, such that

$$
\lim _{m_{3} \rightarrow \infty} \sup _{t>t_{0}} P\left\{\left|x_{0}(t)\right|>m_{3}\right\}=0
$$

Substituting (17) into the subsystem (13a), one gets

$$
\mathrm{d} x_{0}=-p_{1}^{*} \eta_{0} x_{0} \mathrm{~d} t+p_{1}^{*} m_{1} x_{0} \mathrm{~d} B .
$$

Proposition 14. For initial state $x_{0}\left(t_{0}\right) \neq 0$, the solution of (21) will never reach the zero, which avoids the uncontrollability of the subsystem (13b).

Proof. From Lemma 2.3 ([27, Page 93]), the following equality will be the solution of (21):

$$
\begin{gathered}
x_{0}(t)=x_{0}\left(t_{0}\right) \exp \left\{\int_{t_{0}}^{t}\left(-p_{1}^{*} \eta_{0}-\frac{1}{2}\left(p_{1}^{*} m_{1}\right)^{2}\right) \mathrm{d} s\right. \\
\left.+\int_{t_{0}}^{t} p_{1}^{*} m_{1} \mathrm{~d} \omega\right\} .
\end{gathered}
$$


From the above expression of $x_{0}(t)$ and $x_{0}\left(t_{0}\right) \neq 0$, it is easy to see that $x_{0}(t)$ will never cross the origin at the time interval $t \in\left(t_{0},+\infty\right)$.

In the following Section 3.2, the other states will be regulated to the origin in probability by the design of the control input $u$.

3.2. Other States Stabilization. In order to design a smooth adaptive state-feedback controller, the following state-input scaling discontinuous transformation is needed:

$$
z_{1}=\frac{x_{1}}{u_{0}}, \quad z_{2}=x_{2}
$$

Remark 15. For the initial state $x_{0}\left(t_{0}\right) \neq 0$, from Proposition 14, one can obtain that transformation (23) is meaningful.

Under the new $z$-coordinate, the subsystem (13b) is transformed into

$$
\begin{aligned}
\mathrm{d} z_{1}= & p_{1}^{*}\left(-\eta_{0} \mathrm{z}_{2}-0.5 p_{1}^{*} z_{1} \omega_{2}^{2}-p_{1}^{*} z_{2} \frac{\omega_{2}^{2}}{x_{0}^{2}}+\eta_{0} z_{1}\right. \\
& \left.+p_{2}^{*} v_{2} \frac{\omega_{2}}{x_{0}}+p_{1}^{*} z_{1} \frac{\omega_{2}^{2}}{x_{0}^{2}}\right) \mathrm{d} t \\
& +p_{1}^{*}\left(z_{2} \frac{\omega_{2}}{x_{0}}-z_{1} \frac{\omega_{2}}{x_{0}}\right) \mathrm{d} B, \\
\mathrm{~d} z_{2}= & p_{2}^{*} u \mathrm{~d} t+\left(p_{1}^{*} \eta_{0} z_{1} x_{0}^{2}-0.5\left(p_{1}^{*}\right)^{2} z_{2} \omega_{2}^{2}\right) \mathrm{d} t \\
& +\left(p_{2}^{*} v_{2}-p_{1}^{*} z_{1} x_{0} \omega_{2}\right) \mathrm{d} B .
\end{aligned}
$$

To invoke the backstepping method, the error variables $\varepsilon_{1}$ and $\varepsilon_{2}$ are given by

$$
\varepsilon_{1}=z_{1}, \quad \varepsilon_{2}=z_{2}-\alpha_{1}\left(\varepsilon_{1}\right) \text {. }
$$

Step 1. Define the first Lyapunov candidate function:

$$
V_{1}=\frac{1}{4 p_{1}^{*}} \varepsilon_{1}^{4} .
$$

By (24)-(26) and Definition 1, one has

$$
\begin{aligned}
\mathscr{L} V_{1} \leq \varepsilon_{1}^{3}\left\{-\eta_{0} z_{2}-\frac{1}{2} p_{1}^{*} z_{1} \omega_{2}^{2}-p_{1}^{*} z_{2} \frac{\omega_{2}^{2}}{x_{0}^{2}}+\eta_{0} z_{1}\right. \\
\left.+p_{2}^{*} v_{2} \frac{\omega_{2}}{x_{0}}+p_{1}^{*} z_{1} \frac{\omega_{2}^{2}}{x_{0}^{2}}\right\} \\
+3 \varepsilon_{1}^{2}\left\{\left(p_{1}^{*}\right)^{2} z_{2}^{2} \frac{\omega_{2}^{2}}{x_{0}^{2}}+\left(p_{1}^{*}\right)^{2} z_{1}^{2} \frac{\omega_{2}^{2}}{x_{0}^{2}}\right\} .
\end{aligned}
$$

The virtual control can be chosen as

$$
\alpha_{1}\left(\varepsilon_{1}\right)=c_{1} \varepsilon_{1} \text {, }
$$

where $c_{1}$ is a positive constant, which will be chosen later. From (27), Lemma 5, and simple operation, we have the following inequalities:

$$
\begin{gathered}
-\eta_{0} z_{2} \varepsilon_{1}^{3} \leq \eta_{0}\left\{\frac{3 d}{4} \varepsilon_{1}^{4}+\frac{3}{4 d^{3}} \varepsilon_{2}^{4}\right\}-\eta_{0} c_{1} \varepsilon_{1}^{4}, \\
-\frac{1}{2} \varepsilon_{1}^{3} p_{1}^{*} z_{1} \omega_{2}^{2} \leq \frac{1}{2} p_{\max } m_{1}^{2} m_{3}^{2} \varepsilon_{1}^{4}, \\
-p_{1}^{*} \varepsilon_{1}^{3} z_{2} \frac{\omega_{2}^{2}}{x_{0}^{2}} \leq p_{\max } m_{1}^{2}\left\{\frac{3}{4}+c_{1}\right\} \varepsilon_{1}^{4}+\frac{1}{4} p_{\max } m_{1}^{2} \varepsilon_{2}^{4}, \\
p_{2}^{*} v_{2} \frac{\omega_{2}}{x_{0}} \leq \frac{1}{4} \varepsilon_{1}^{4}+\frac{3}{4}\left(m_{1} m_{2}\right)^{4 / 3}\left(p_{2}^{*}\right)^{4 / 3} \varepsilon_{2}^{4}, \\
3\left(p_{1}^{*}\right)^{2} \varepsilon_{1}^{3} z_{2}^{2} \frac{\omega_{2}^{2}}{x_{0}^{2}} \leq 3 p_{\max }^{2} z_{1} \frac{\omega_{2}^{2}}{x_{0}^{2}} \leq p_{\max } m_{1}^{2} \varepsilon_{1}^{4}, \\
3\left(p_{1}^{2}+1\right\} \varepsilon_{1}^{4}+3 p_{\max }^{2} \varepsilon_{1}^{2} z_{1}^{2} \frac{\omega_{2}^{2}}{x_{0}^{2}} \leq 3 p_{\max }^{2} m_{1}^{2} \varepsilon_{1}^{4},
\end{gathered}
$$

where $d>0$ is a design parameter. Substituting these above inequalities into (27), it is easy to see that

$$
\begin{gathered}
\mathscr{L} V_{1} \leq\left\{-\eta_{0} c_{1} e+\left(1+\frac{3 d}{4}\right) \eta_{0}-c_{1} \eta_{0}(1-e)\right. \\
+\frac{p_{\max } m_{1}^{2}\left(2 m_{3}^{2}+4 c_{1}+24 p_{\max } c_{1}^{2}\right)}{4} \\
\left.+\frac{p_{\max } m_{1}^{2}\left(36 p_{\max }+7\right)+1}{4}\right\} \varepsilon_{1}^{4} \\
+\left\{\frac{\eta_{0}}{4 d^{3}}+\frac{p_{\max } m_{1}^{2}}{4}+3 p_{\max }^{2} m_{1}^{2}\right\} \varepsilon_{2}^{4} \\
+\frac{3}{4}\left(m_{1} m_{2}\right)^{4 / 3}\left(p_{2}^{*}\right)^{4 / 3} \varepsilon_{2}^{4},
\end{gathered}
$$

where $e$ is a design parameter and $0<e<1$. If we select parameters $\eta_{0}$ and $c_{1}$ to satisfy

$$
\begin{gathered}
c_{1} \geq \frac{4+3 d}{4 e}, \\
\eta_{0} \geq \frac{p_{\max } m_{1}^{2}\left(2 m_{3}^{2}+4 c_{1}+24 p_{\max } c_{1}^{2}+36 p_{\max }+7\right)+1}{2 c_{1}(1-e)},
\end{gathered}
$$

one has

$$
\begin{aligned}
\mathscr{L} V_{1} \leq & -\frac{c_{1} \eta_{0}(1-e)}{2} \varepsilon_{1}^{4}+\frac{3}{4}\left(m_{1} m_{2}\right)^{4 / 3}\left(p_{2}^{*}\right)^{4 / 3} \varepsilon_{2}^{4} \\
& +\left\{\frac{\eta_{0}}{4 d^{3}}+\frac{p_{\max } m_{1}^{2}}{4}+3 p_{\max }^{2} m_{1}^{2}\right\} \varepsilon_{2}^{4} .
\end{aligned}
$$


Step 2. By (24), (25), (32), and Itô formula (Theorem 6.2, [27, Page 32]), one gets

$$
\begin{aligned}
\mathrm{d} \varepsilon_{2}=p_{2}^{*} u \mathrm{~d} t+ & \left\{p_{1}^{*} \eta_{0} z_{1} x_{0}^{2}-\frac{1}{2}\left(p_{1}^{*}\right)^{2} z_{2} \omega_{2}^{2}\right. \\
& +p_{1}^{*} c_{1} \eta_{0} z_{2}+\frac{1}{2}\left(p_{1}^{*}\right)^{2} c_{1} z_{1} \omega_{2}^{2} \\
& -p_{1}^{*} c_{1} \eta_{0} z_{1}+\left(p_{1}^{*}\right)^{2} c_{1} z_{2} \frac{\omega_{2}^{2}}{x_{0}^{2}} \\
& \left.-p_{1}^{*} p_{2}^{*} c_{1} v_{2} \frac{\omega_{2}}{x_{0}}-\left(p_{1}^{*}\right)^{2} c_{1} z_{1} \frac{\omega_{2}^{2}}{x_{0}^{2}}\right\} \mathrm{d} t \\
+\left(p_{2}^{*} v_{2}-\right. & \left.p_{1}^{*} z_{1} x_{0} \omega_{2}-p_{1}^{*} c_{1} z_{2} \frac{\omega_{2}}{x_{0}}+p_{1}^{*} c_{1} z_{1} \frac{\omega_{2}}{x_{0}}\right) \mathrm{d} B
\end{aligned}
$$
ter

To deal with the uncertain parameter $p_{2}^{*}$, define parame-

$$
\Theta=\max \left\{p_{2}^{*},\left(p_{2}^{*}\right)^{4 / 3}, \frac{1}{p_{2}^{*}},\left(\frac{1}{p_{2}^{*}}\right)^{4 / 3},\left(\frac{1}{p_{2}^{*}}\right)^{2}\right\},
$$

and $\widetilde{\Theta}=\Theta-\widehat{\Theta}$ being the parameter estimation error, $\widehat{\Theta}$ being the estimate of $\Theta$. Define the second Lyapunov candidate function:

$$
V_{2}=V_{1}+\frac{1}{4 p_{2}^{*}} \varepsilon_{2}^{4}+\frac{1}{2} \widetilde{\Theta}^{2} .
$$

From (33), (35), and Definition 1, one can obtain

$$
\begin{aligned}
& \mathscr{L} V_{2} \leq-\frac{c_{1} \eta_{0}(1-e)}{2} \varepsilon_{1}^{4}+\frac{3}{4}\left(m_{1} m_{2}\right)^{4 / 3}\left(p_{2}^{*}\right)^{4 / 3} \varepsilon_{2}^{4} \\
&+\left\{\frac{\eta_{0}}{4 d^{3}}+\frac{p_{\max } m_{1}^{2}}{4}+3 p_{\max }^{2} m_{1}^{2}\right\} \varepsilon_{2}^{4} \\
&+\varepsilon_{2}^{3}\left\{u+\frac{p_{1}^{*}}{p_{2}^{*}} \eta_{0} z_{1} x_{0}^{2}-\frac{1}{2} \frac{\left(p_{1}^{*}\right)^{2}}{p_{2}^{*}} z_{2} \omega_{2}^{2}\right. \\
&+\frac{p_{1}^{*}}{p_{2}^{*}} c_{1} \eta_{0} z_{2}+\frac{1}{2} \frac{\left(p_{1}^{*}\right)^{2}}{p_{2}^{*}} c_{1} z_{1} \omega_{2}^{2} \\
&-\frac{p_{1}^{*}}{p_{2}^{*}} c_{1} \eta_{0} z_{1}+\frac{\left(p_{1}^{*}\right)^{2}}{p_{2}^{*}} c_{1} z_{2} \frac{\omega_{2}^{2}}{x_{0}^{2}} \\
&\left.-p_{1}^{*} c_{1} v_{2} \frac{\omega_{2}}{x_{0}}-\frac{\left(p_{1}^{*}\right)^{2}}{p_{2}^{*}} c_{1} z_{1} \frac{\omega_{2}^{2}}{x_{0}^{2}}\right\} \mathrm{d} t \\
&+6 \varepsilon_{2}^{2}\left\{\begin{array}{c}
p_{2}^{*} v_{2}^{2}+\frac{\left(p_{1}^{*}\right)^{2}}{p_{2}^{*}} z_{1}^{2} x_{0}^{2} \omega_{2}^{2} \\
\left.+\frac{\left(p_{1}^{*}\right)^{2}}{p_{2}^{*}} c_{1}^{2} z_{2}^{2} \frac{\omega_{2}^{2}}{x_{0}^{2}}+\frac{\left(p_{1}^{*}\right)^{2}}{p_{2}^{*}} c_{1}^{2} z_{1}^{2} \frac{\omega_{2}^{2}}{x_{0}^{2}}\right\}
\end{array}\right.
\end{aligned}
$$

$-\widetilde{\Theta} \widehat{\Theta}$
By (34), (36), and Lemma 5, we have the following inequalities:

$$
\begin{aligned}
& \frac{p_{1}^{*}}{p_{2}^{*}} \eta_{0} z_{1} x_{0}^{2} \varepsilon_{2}^{3} \leq \Theta \frac{3}{4}\left(p_{\max } \eta_{0} m_{3}^{2}\right)^{4 / 3} \varepsilon_{2}^{4}+\frac{1}{4} \varepsilon_{1}^{4}, \\
& -\frac{1}{2} \frac{\left(p_{1}^{*}\right)^{2}}{p_{2}^{*}} z_{2} \omega_{2}^{2} \varepsilon_{2}^{4} \\
& \leq \Theta\left\{\frac{1}{2} p_{\max }^{2} m_{3}^{2} m_{1}^{2}+\frac{3}{8}\left(c_{1} p_{\max }^{2} m_{3}^{2} m_{1}^{2}\right)^{4 / 3}\right\} \varepsilon_{2}^{4}+\frac{1}{8} \varepsilon_{1}^{4}, \\
& \frac{p_{1}^{*}}{p_{2}^{*}} c_{1} \eta_{0} z_{2} \varepsilon_{2}^{3} \leq \Theta\left\{c_{1} p_{\max } \eta_{0}+\frac{3}{4} c_{1}^{8 / 3} p_{\max }^{4 / 3} \eta_{0}^{4 / 3}\right\} \varepsilon_{2}^{4}+\frac{1}{4} \varepsilon_{1}^{4}, \\
& \frac{1}{2} \frac{\left(p_{1}^{*}\right)^{2}}{p_{2}^{*}} c_{1} z_{1} \omega_{2}^{2} \varepsilon_{2}^{3} \leq \Theta \frac{3}{8}\left(c_{1} p_{\max }^{2} m_{3}^{2} m_{1}^{2}\right)^{4 / 3} \varepsilon_{2}^{4}+\frac{1}{8} \varepsilon_{1}^{4}, \\
& -\frac{p_{1}^{*}}{p_{2}^{*}} c_{1} \eta_{0} z_{1} \varepsilon_{2}^{3} \leq \Theta \frac{3}{4}\left(c_{1} \eta_{0} p_{\max }\right)^{4 / 3} \varepsilon_{2}^{4}+\frac{1}{4} \varepsilon_{1}^{4}, \\
& \frac{\left(p_{1}^{*}\right)^{2}}{p_{2}^{*}} c_{1} z_{2} \frac{\omega_{2}^{2}}{x_{0}^{2}} \varepsilon_{2}^{3} \leq \Theta c_{1} p_{\max }^{2} m_{1}^{2} \varepsilon_{2}^{4}+\Theta \frac{3}{4}\left(c_{1}^{2} m_{1}^{2} p_{\max }^{2}\right)^{4 / 3} \varepsilon_{2}^{4} \\
& +\frac{1}{4} \varepsilon_{1}^{4} \\
& -p_{1}^{*} c_{1} v_{2} \frac{\omega_{2}}{x_{0}} \varepsilon_{2}^{3} \leq p_{\max } m_{1} m_{2} c_{1} \varepsilon_{2}^{4}, \\
& -\frac{\left(p_{1}^{*}\right)^{2}}{p_{2}^{*}} c_{1} z_{1} \frac{\omega_{2}^{2}}{x_{0}^{2}} \varepsilon_{2}^{3} \leq \frac{1}{4} \varepsilon_{1}^{4}+\Theta \frac{3}{4}\left(p_{\max }^{2} c_{1} m_{1}^{2}\right)^{4 / 3} \varepsilon_{2}^{4}, \\
& 6 \varepsilon_{2}^{2}\left\{p_{2}^{*} v_{2}^{2} z_{1}^{2} x_{0}^{2}+\frac{\left(p_{1}^{*}\right)^{2}}{p_{2}^{*}} \omega_{2}^{2}+\frac{\left(p_{1}^{*}\right)^{2}}{p_{2}^{*}} c_{1}^{2} z_{2}^{2} \frac{\omega_{2}^{2}}{x_{0}^{2}}\right. \\
& \left.+\frac{\left(p_{1}^{*}\right)^{2}}{p_{2}^{*}} c_{1}^{2} z_{1}^{2} \frac{\omega_{2}^{2}}{x_{0}^{2}}\right\} \\
& \leq \Theta\left\{6 m_{2}^{2}+3 p_{\max }^{4} m_{3}^{8} m_{1}^{4}+12 p_{\max }^{2} m_{1}^{2}\right. \\
& \left.+6 p_{\max }^{4} m_{1}^{4} c_{1}^{4}+3 p_{\max }^{4} m_{1}^{4} c_{1}^{4}\right\} \varepsilon_{2}^{4}+12 \varepsilon_{1}^{4} .
\end{aligned}
$$

Substituting the above inequalities into (36) and adding and subtracting the term $c_{2} \varepsilon_{2}^{4}$ on the right-hand side of (36), we have

$$
\begin{aligned}
\mathscr{L} V_{2} \leq & -\left\{\frac{c_{1} \eta_{0}(1-e)}{2}-13.5\right\} \varepsilon_{1}^{4}-c_{2} \varepsilon_{2}^{4} \\
& +H_{21} \varepsilon_{2}^{4}+\varepsilon_{2}^{3} u+\Theta H_{22} \varepsilon_{2}^{4}-\widetilde{\Theta} \dot{\Theta} \\
\leq & -\bar{c}_{1} \varepsilon_{1}^{4}-c_{2} \varepsilon_{2}^{4}+\varepsilon_{2}^{3} u+\widetilde{\Theta}\left\{H_{22} \varepsilon_{2}^{4}-\dot{\widehat{\Theta}}\right\} \\
& +\left\{H_{21}+\sqrt{1+\widehat{\Theta}^{2}} H_{22}\right\} \varepsilon_{2}^{4},
\end{aligned}
$$


where

$$
\begin{aligned}
H_{21}= & \frac{\eta_{0}}{4 d^{3}}+\frac{p_{\max } m_{1}^{2}}{4}+3 p_{\max }^{2} m_{1}^{2}+p_{\max } m_{1} m_{2} c_{1}, \\
H_{22}= & c_{2}+\frac{3}{4}\left(p_{\max } \eta_{0} m_{3}^{2}\right)^{4 / 3}+\frac{1}{2} p_{\max }^{2} m_{3}^{2} m_{1}^{2} \\
& +c_{1} p_{\max } \eta_{0}+\frac{3}{8}\left(c_{1} p_{\max }^{2} m_{3}^{2} m_{1}^{2}\right)^{4 / 3} \\
& +\frac{3}{4} c_{1}^{8 / 3} p_{\max }^{4 / 3} \eta_{0}^{4 / 3}+\frac{3}{8}\left(c_{1} p_{\max }^{2} m_{3}^{2} m_{1}^{2}\right)^{4 / 3} \\
& +\frac{3}{4}\left(c_{1} \eta_{0} p_{\max }\right)^{4 / 3}+c_{1} p_{\max }^{2} m_{1}^{2} \\
& +\frac{3}{4}\left(c_{1}^{2} m_{1}^{2} p_{\max }^{2}\right)^{4 / 3}+6 m_{2}^{2}+12 p_{\max }^{2} m_{1}^{2} \\
& +3 p_{\max }^{4} m_{3}^{8} m_{1}^{4}+6 p_{\max }^{4} m_{1}^{4} c_{1}^{4}+3 p_{\max }^{4} m_{1}^{4} c_{1}^{4} \\
& +\frac{3}{4}\left(p_{\max }^{3} c_{1} m_{1}^{2}\right)^{4 / 3}+\frac{3}{4}\left(m_{1} m_{2}\right)^{4 / 3} .
\end{aligned}
$$

One can choose the actual control law $u$ and the adaptive laws $\widehat{\Theta}$ as follows:

$$
\begin{gathered}
u=-\left\{H_{21}+\sqrt{1+\widehat{\Theta}^{2}} H_{22}\right\} \varepsilon_{2}, \\
\dot{\bar{\Theta}}=H_{22} \varepsilon_{2}^{4} .
\end{gathered}
$$

Substituting (40) into (38), one gets

$$
\mathscr{L} V_{2} \leq-\bar{c}_{1} \varepsilon_{1}^{4}-c_{2} \varepsilon_{2}^{4}
$$

Choosing the Lyapunov function as

$$
V=V_{0}+V_{2}
$$

together with (19) and (41), we have

$$
\mathscr{L} V \leq-\lambda x_{0}^{2}-\bar{c}_{1} \varepsilon_{1}^{4}-c_{2} \varepsilon_{2}^{4} .
$$

Theorem 16. If Assumptions 9 and 10 hold, one can choose positive constants $\lambda, m_{1}, m_{2}, m_{3}$, and $p_{\max }$, with $d>0$ and $0<e<1$ satisfying $\bar{c}_{1}>0$ and (31); for positive constant $c_{2}$, one has the following.

(i) The closed-loop system composed by (13a), (17), (24), and (40) has an almost surely unique solution on $\left[t_{0}, \infty\right)$ for $\forall x_{0}\left(t_{0}\right), z\left(t_{0}\right)$ and $\widehat{\Theta}\left(t_{0}\right)$.

(ii) The equilibrium $\left(x_{0}, z, \widetilde{\Theta}\right)=(0,0,0)$ of the closed-loop system is globally stable in probability.

(iii) For initial condition $\forall x_{0}\left(t_{0}\right), z\left(t_{0}\right)$, and $\widehat{\Theta}\left(t_{0}\right)$, $P\left\{\lim _{t \rightarrow \infty}\left(\left|x_{0}(t)\right|+|z(t)|\right)=0\right\}=1$, $P\left\{\lim _{t \rightarrow \infty} \widehat{\Theta}(t)\right.$ exists and is finite $\}=1$, where $\widetilde{\Theta}=\Theta-\widetilde{\Theta}$ and $z(t)=\left(z_{1}(t), z_{2}(t)\right)$.

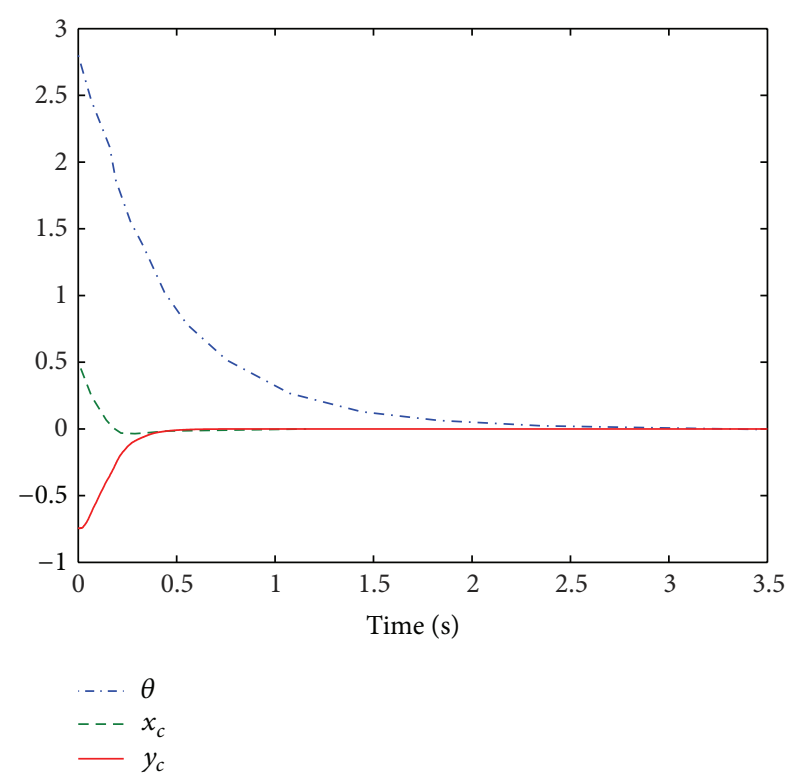

Figure 1: The responses of states $\theta, x_{c}$, and $y_{c}$ with respect to time.

Proof. From conditions in Theorem 16, it is easy to see that constants $\lambda>0, \bar{c}_{1}>0$, and $c_{2}>0$. So, $\mathscr{L} V$ in (43) becomes the same form as (3.19) in [21]. Using (43) and Lemma 4, Theorem 16 can be proved.

\section{Switching Control Stability}

In Section 2, the case of $x_{0}\left(t_{0}\right) \neq 0$ is discussed. We design controllers $u_{0}$ and $u$ for systems (13a) and (13b) as in (17) and (40), respectively. Now we turn to the case of $x_{0}\left(t_{0}\right)=0$. When the initial $x_{0}\left(t_{0}\right)=0$, one can choose an open loop control $u_{0}=-u_{0}^{*} \neq 0$ to drive the state $x_{0}$ away from zero in a limited time.

In fact, when we choose an open loop control $u_{0}=$ $-u_{0}^{*} \neq 0$, system (13a) will be in the following form:

$$
\mathrm{d} x_{0}=-u_{0}^{*} \mathrm{~d} t+p_{1}^{*} \omega_{2}\left(x_{0}\right) \mathrm{d} B .
$$

For a given constant $l>0$, define a stopping time $\tau_{l}=\inf \{t$ : $\left.t \geq t_{0},\left|x_{0}(t)\right| \geq l\right\}$. With the similar analysis in Section $\mathrm{V}$ in [22], we have $P\left(\tau_{l}-t_{0} \geq T\right) \leq l / T u_{0}^{*}$, which means that $P\left(\tau_{l}=\infty\right)=0$ for any $l>0$. Letting $t_{s}^{*}=\tau_{l}$, it is easy to see that

$$
\left|x_{0}\left(t_{s}^{*}\right)\right|=\left|x_{0}\left(\tau_{l}\right)\right|=l \neq 0 .
$$

So, there exists $t_{s}^{*}>0$, such that $x_{0}\left(t_{s}^{*}\right) \neq 0$. After that, at the time $t=t_{s}^{*}$, we switch the control inputs $u_{0}$ and $u$ to (17) and (40) in $t \in\left[t_{s}^{*},+\infty\right)$, respectively.

Theorem 17. If Assumptions 9 and 10 hold, one can apply the following switching control procedure to system (11):

(i) when the initial state belongs to

$$
\left\{\left(\theta\left(t_{0}\right), x_{c}\left(t_{0}\right), y_{c}\left(t_{0}\right)\right) \in \mathbb{R}^{3} \mid \theta\left(t_{0}\right) \neq 0\right\},
$$



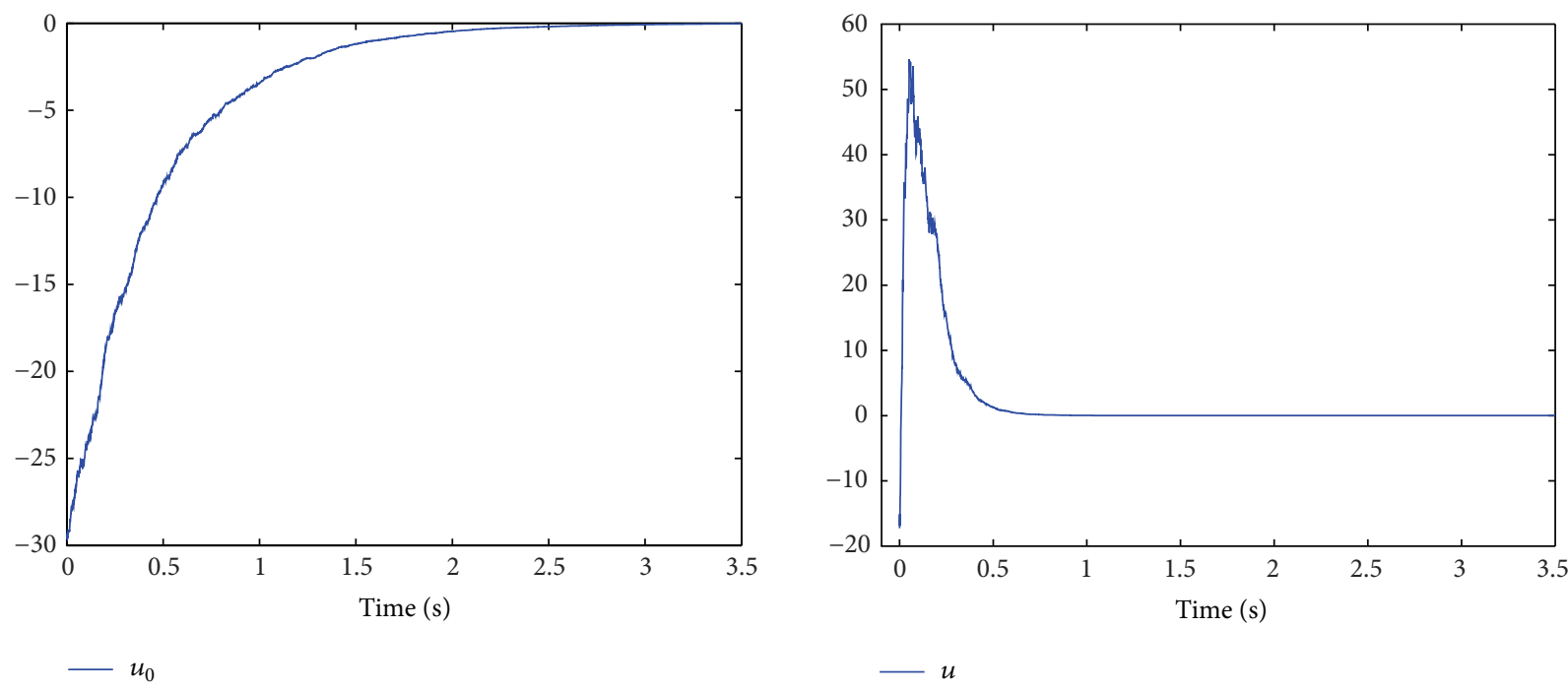

FIGURE 2: The responses of controllers $u_{0}$ and $u$ with respect to time.

(ii) when the initial state belongs to

$$
\left\{\left(\theta\left(t_{0}\right), x_{c}\left(t_{0}\right), y_{c}\left(t_{0}\right)\right) \in \mathbb{R}^{3} \mid \theta\left(t_{0}\right)=0\right\} .
$$

One designs control inputs $u_{0}$ and $u$ in form (17) and (40), respectively; for $t \in\left[t_{0}, t_{s}^{*}\right)$, one can choose the control law $u_{0}=-u_{0}^{*} \neq 0$ and $u=u^{*}$; for $t \in\left[t_{s}^{*},+\infty\right)$, at the time $t=t_{s}^{*}$, one switches the control inputs $u_{0}$ and $u$ to (17) and (40), respectively.

Then, for any initial condition in the state space, the states of system (11) are asymptotically regulated to zero in probability.

Proof. Firstly, we consider the case that the initial state belongs to

$$
\left\{\left(\theta\left(t_{0}\right), x_{c}\left(t_{0}\right), y_{c}\left(t_{0}\right)\right) \in \mathbb{R}^{3} \mid \theta\left(t_{0}\right) \neq 0\right\} .
$$

From Theorems 12 and 16, for the closed-loop system composed by (13a), (17), (24), and (40), states $x_{0}$ and $z(t)$ are regulated to zero in probability, $\widehat{\Theta}(t)$ is bounded in probability, and $P\left\{\lim _{t \rightarrow \infty}\left(\left|x_{0}(t)\right|+|z(t)|\right)=0\right\}=1$. This implies that states $x_{0}$ and $z(t)$ are globally asymptotically regulated to zero in probability and bounded in probability. As a result of (23), one gets that the states $x_{0}, x_{1}$, and $x_{2}$ of closedloop system composed by (11), (17), and (40) asymptotically converge to zero in probability and all bounded in probability. By orthogonal transformation (12), one can obtain that the states $\theta, x_{c}$, and $y_{c}$ of closed-loop system composed by (11), (17), and (40) are asymptotically stabilized in probability.

Secondly, when the initial state belongs to

$$
\left\{\left(\theta\left(t_{0}\right), x_{c}\left(t_{0}\right), y_{c}\left(t_{0}\right)\right) \in \mathbb{R}^{3} \mid \theta\left(t_{0}\right)=0\right\},
$$

we use the constant control $u_{0}=-u_{0}^{*} \neq 0$ in order to drive $x_{0}$ far away from the origin, which guarantees that all the signals are bounded in probability during $\left[t_{0}, t_{s}^{*}\right)$. Then, in view of $x_{0}\left(t_{s}^{*}\right) \neq 0$, the switching control strategy is applied to system (11) at the time instant $t_{s}^{*}>0$. This completes the proof.

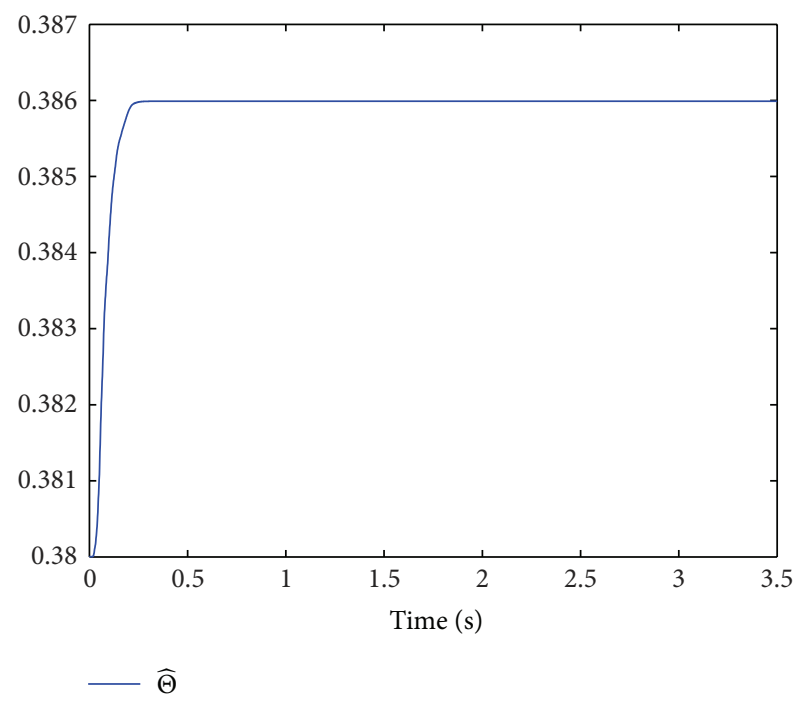

FIGURE 3: The response of estimate parameter $\widehat{\Theta}$ with respect to time.

\section{A Simulation Example}

Consider the system (11) with $\omega_{2}=0.5 \theta$ and $v_{2}=x_{2}-$ $\left(3.5 x_{1} / u_{0}\right)$. In simulation, one can choose $p_{1}^{*}=0.2, p_{2}^{*}=0.1$, $e=0.5, p_{\max }=0.25, d=1, c_{1}=5.5, c_{2}=0.2, \eta_{0}=10.5$, $m_{1}=0.5, m_{2}=1$, and $m_{3}=3$ and the initial values $\theta(0)=2.8$, $x_{c}(0)=-0.678, y_{c}(0)=0.528$, and $\widehat{\Theta}(0)=0.38$. Figures 1,2 , and 3 give the responses of the closed-loop system consisting of (11), (17), and (40).

From Figure 1, it is easy to see that the states $\theta, x_{c}$, and $y_{c}$ are asymptotically regulated to zero in probability in spite of the stochastic disturbances. As shown in Figure 2, the control inputs $u_{0}$ and $u$ are convergent to a small neighborhood of zero asymptotically. Figure 3 indicates that the estimated parameter $\widehat{\Theta}$ is bounded. 


\section{Conclusions}

In this paper, we extend the nonholonomic mobile robots with unknown parameters to the stochastic case. Based on backstepping technique, adaptive state-feedback stabilizing controllers are designed for stochastic nonholonomic mobile robots with unknown parameters. A switching control strategy for the original system is given, which guarantees that the states of closed-loop system are asymptotically stabilized at the zero equilibrium point in probability.

There exist some problems to be discussed, for example, how to design the controller for the dynamic stochastic nonholonomic systems with unknown parameters.

\section{Acknowledgments}

The authors would like to express sincere gratitude to the editor and reviewers' hard work. This paper is supported by the National Science Foundation (no. 61304004) and Natural Science Foundation of Hebei Province of China (no. A2014106035), Doctoral Natural Science Foundation of Shijiazhaung University.

\section{References}

[1] R. W. Brockett, "Asymptotic stability and feedback stabilization," in Differential Geometric Control Theory (Houghton, Mich., 1982), vol. 27 of Progr. Math., pp. 181-191, Birkhäuser, Boston, Mass, USA, 1983.

[2] Z.-P. Jiang, "Iterative design of time-varying stabilizers for multi-input systems in chained form," Systems \& Control Letters, vol. 28, no. 5, pp. 255-262, 1996.

[3] S. S. Ge, Z. Wang, and T. H. Lee, "Adaptive stabilization of uncertain nonholonomic systems by state and output feedback," Automatica, vol. 39, no. 8, pp. 1451-1460, 2003.

[4] Q.-D. Wang and C.-L. Wei, "Robust adaptive control of nonholonomic systems with nonlinear parameterization," Acta Automatica Sinica, vol. 33, no. 4, pp. 399-403, 2007.

[5] Y.-P. Tian and S. Li, "Exponential stabilization of nonholonomic dynamic systems by smooth time-varying control," Automatica, vol. 38, no. 8, pp. 1139-1146, 2002.

[6] K.-C. Cao, "Global $\mathscr{K}$-exponential trackers for nonholonomic chained-form systems based on LMI," International Journal of Systems Science, vol. 42, no. 12, pp. 1981-1992, 2011.

[7] G. Campion, G. Bastin, and B. D’Andréa-Novel, "Structural properties and classification of kinematic and dynamic models of wheeled mobile robots," IEEE Transactions on Robotics and Automation, vol. 12, no. 1, pp. 47-62, 1996.

[8] C. Wang, Y. Mei, Z. Liang, and Q. Jia, "Dynamic feedback tracking control of non-holonomic mobile robots with unknown camera parameters," Transactions of the Institute of Measurement and Control, vol. 32, no. 2, pp. 155-169, 2010.

[9] Z.-Y. Liang and C.-L. Wang, "Robust stabilization of nonholonomic chained form systems with uncertainties," Acta Automatica Sinica, vol. 37, no. 2, pp. 129-142, 2011.

[10] F. Yang and C.-L. Wang, "Adaptive stabilization for uncertain nonholonomic dynamic mobile robots based on visual servoing feedback," Acta Automatica Sinica, vol. 37, no. 7, pp. 857-864, 2011.

[11] J. P. Hespanha, D. Liberzon, and A. S. Morse, "Towards the supervisory control of uncertain nonholonomic systems," in Proceedings of the American Control Conference (ACC '99), pp. 3520-3524, San Diego, Calif, USA, June 1999.

[12] Z.-P. Jiang, "Robust exponential regulation of nonholonomic systems with uncertainties," Automatica, vol. 36, no. 2, pp. 189209, 2000.

[13] Z. Xi, G. Feng, Z. P. Jiang, and D. Cheng, "A switching algorithm for global exponential stabilization of uncertain chained systems," IEEE Transactions on Automatic Control, vol. 48, no. 10, pp. 1793-1798, 2003, New directions on nonlinear control.

[14] G. Wei, Z. Wang, and H. Shu, "Robust filtering with stochastic nonlinearities and multiple missing measurements," Automatica, vol. 45, no. 3, pp. 836-841, 2009.

[15] G. Wei, Z. Wang, J. Lam, K. Fraser, G. P. Rao, and X. Liu, "Robust filtering for stochastic genetic regulatory networks with timevarying delay," Mathematical Biosciences, vol. 220, no. 2, pp. 7380, 2009.

[16] M. Krstić and H. Deng, Stability of Nonlinear Uncertain Systems, Springer, New York, NY, USA, 1998.

[17] Z. Pan and T. Başar, "Backstepping controller design for nonlinear stochastic systems under a risk-sensitive cost criterion," SIAM Journal on Control and Optimization, vol. 37, no. 3, pp. 957-995, 1999.

[18] J. Wang, H. Gao, and H. Li, "Adaptive robust control of nonholonomic systems with stochastic disturbances," Science in China. Series F. Information Sciences, vol. 49, no. 2, pp. 189-207, 2006.

[19] Y. Zhao, J. Yu, and Y. Wu, "State-feedback stabilization for a class of more general high order stochastic nonholonomic systems," International Journal of Adaptive Control and Signal Processing, vol. 25, no. 8, pp. 687-706, 2011.

[20] D. Zhang, C. Wang, H. Chen, F. Yang, and J. Du, "Adaptive stabilization of stochastic non-holonomic systems with nonhomogeneous uncertainties," Transactions of the Institute of Measurement and Control, 2011.

[21] D. Zhang, C. Wang, and H. Chen, "Adaptive state-feedback stabilization for stochastic nonholonomic chained systems," Control Theory \& Applications, vol. 29, no. 11, pp. 1479-1487, 2012.

[22] Z. J. Wu and Y. H. Liu, "Stochastic stabilization of nonholonomic mobile robot with heading-angle-dependent disturbance," Mathematical Problems in Engineering, vol. 2012, Article ID 870498, 17 pages, 2012.

[23] Y. Shang and H. Meng, "Exponential stabilization of nonholonomic mobile robots subject to stochastic disturbance," Journal of Information \& Computational Science, vol. 9, no. 9, pp. 26352642, 2012.

[24] H. Deng, M. Krstić, and R. J. Williams, "Stabilization of stochastic nonlinear systems driven by noise of unknown covariance," IEEE Transactions on Automatic Control, vol. 46, no. 8, pp. 12371253, 2001.

[25] R. Z. Has'minskiǔ, Stochastic Stability of Differential Equations, vol. 7 of Monographs and Textbooks on Mechanics of Solids and Fluids: Mechanics and Analysis, Sijthoff \& Noordhoff, Alphen aan den Rijn, The Netherlands, 1980.

[26] W. Lin and C. Qian, "Adaptive control of nonlinearly parameterized systems: a nonsmooth feedback framework," IEEE 
Transactions on Automatic Control, vol. 47, no. 5, pp. 757-774, 2002.

[27] X. Mao, Stochastic Differential Equations and Their Applications, Horwood Publishing Series in Mathematics \& Applications, Horwood, Chichester, UK, 1997. 


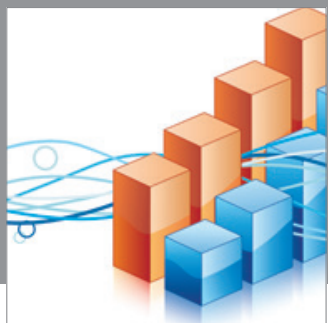

Advances in

Operations Research

mansans

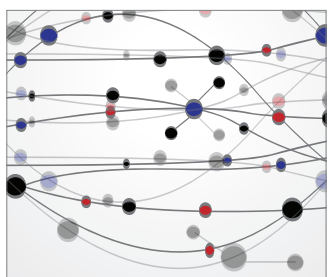

The Scientific World Journal
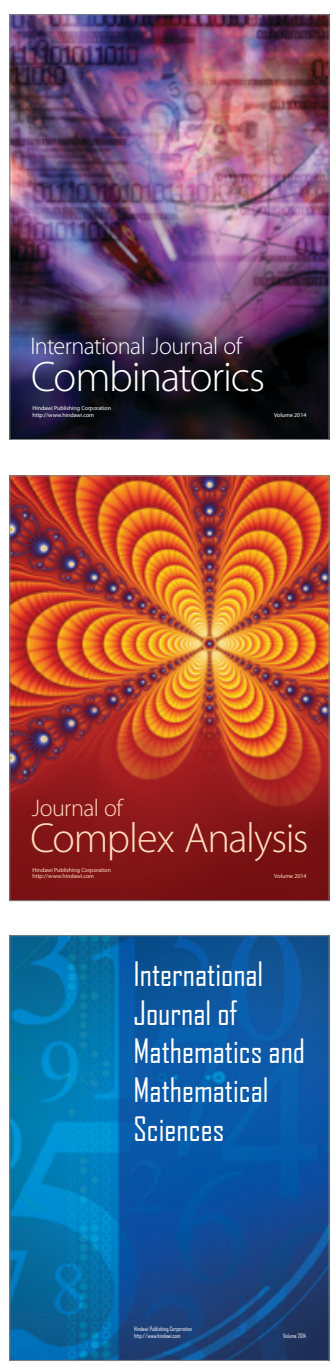
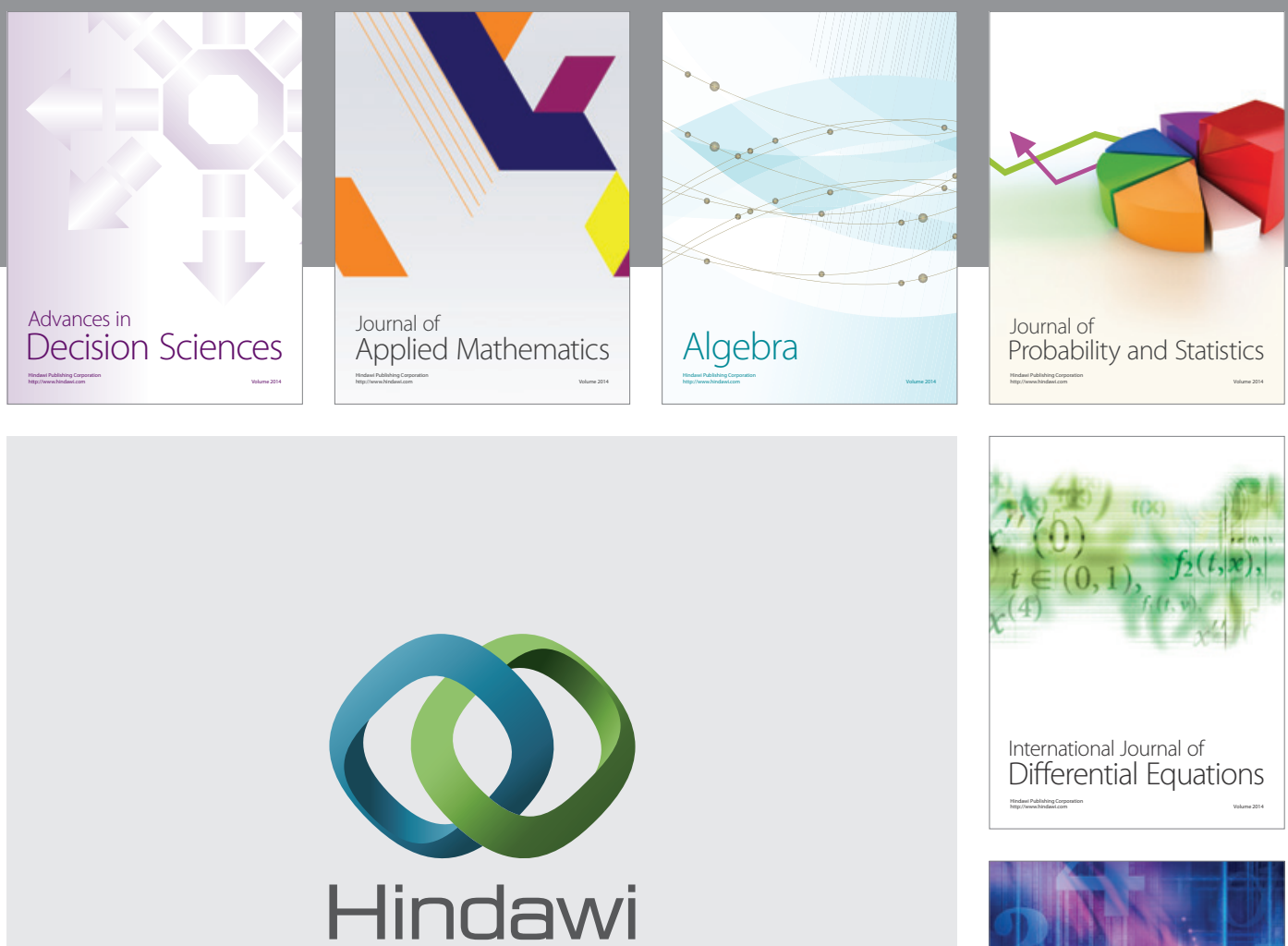

Submit your manuscripts at http://www.hindawi.com
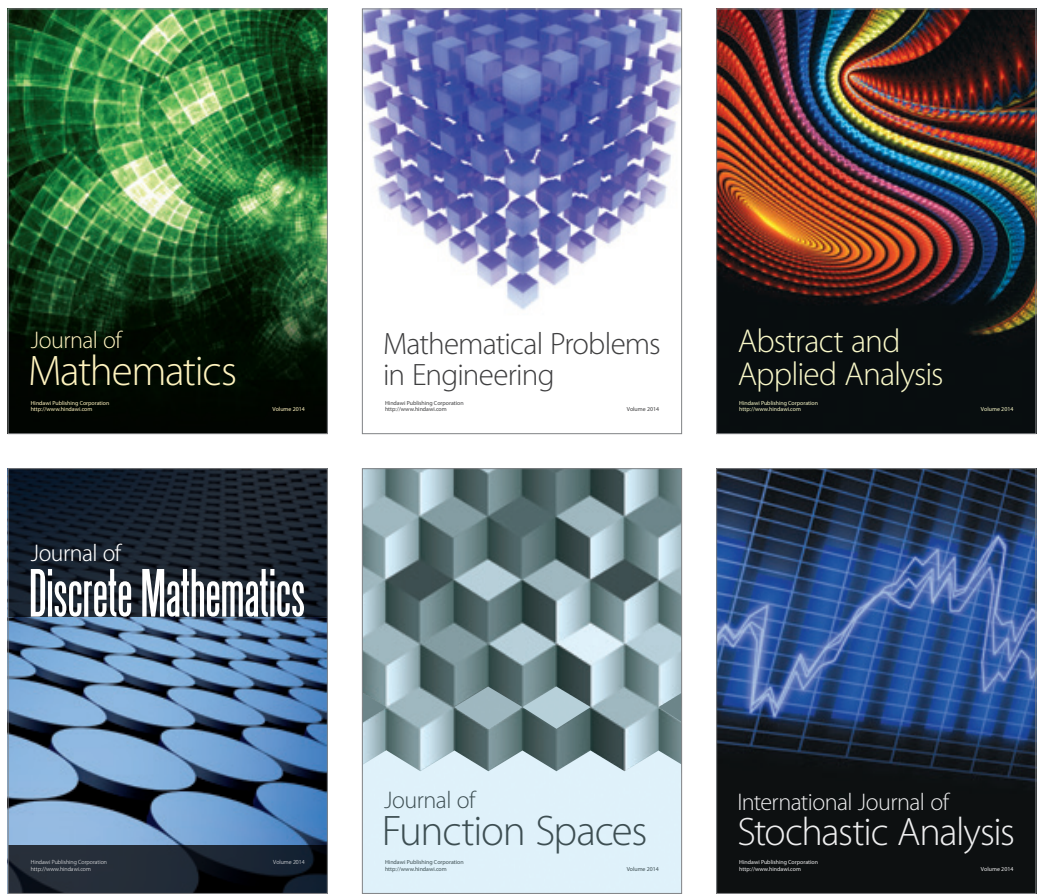

Journal of

Function Spaces

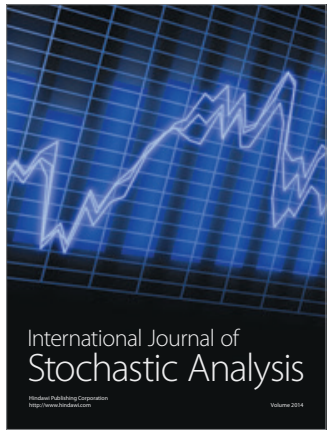

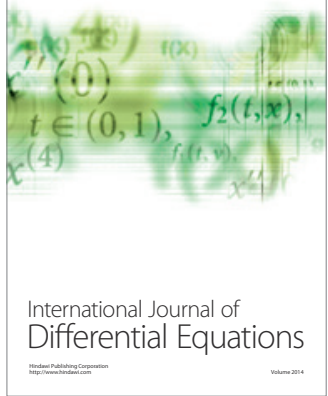
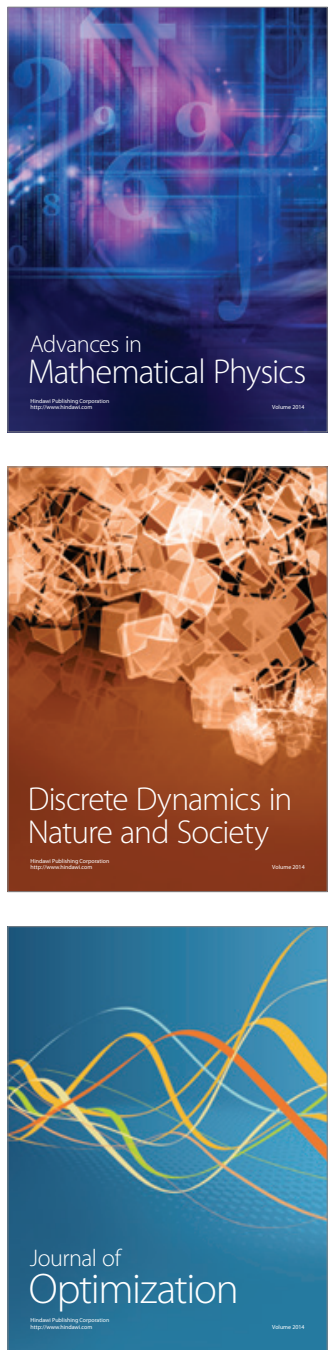